$\underline{\text { Supporting information }}$

\title{
Combined Experimental and Theoretical Insights into Energy Storage Applications of a $\mathrm{VO}_{2}(\mathrm{D})$-Graphene Hybrid
}

\author{
Swagatika Kamila, ${ }^{\text {a,b }}$ Brahmananda Chakraborty, ${ }^{\text {* }}$ Suddhasatwa Basu,,${ }^{\text {a,b }}$ Bikash Kumar \\ Jena, ${ }^{a, b *}$ \\ a.Materials Chemistry Department, CSIR-Institute of Minerals and Materials Technology, \\ Bhubaneswar-751013, India \\ ${ }^{\mathrm{b}}$ Academy of Scientific \& Innovative Research, New Delhi-110001, India \\ ${ }^{\mathrm{c}}$ High Pressure \& Synchrotron Radiation Physics Division, BARC, Mumbai-85
}




\section{Electrochemical Calculations:}

In the case of symmetric supercapacitor device, the specific capacitance of electrode material has been calculated from $\mathrm{CV}$ plot, by following equation $1 .^{1}$

$$
C s p=2 \int I(V) d V / v m V
$$

Where $\int \mathrm{I}(\mathrm{V}) \mathrm{dV}$ is the integrated surface area obtained under the CV plot, $\mathrm{m}$ is the mass of the electrode material, $v$ is the scan rate, and $\mathrm{V}$ is the working potential.

In the case of symmetric supercapacitor device, the specific capacitance has been calculated from GCD plot, by following equation $2 .^{2}$

$$
C_{s p}=4 \frac{I}{m(d V / d t)}
$$

Where $\mathrm{m}$ is the total mass of electrode materials and $\mathrm{dV} / \mathrm{dt}$ is the slope of the discharge curve at constant current 'I'.

For the asymmetric device fabrication, it is necessary to charge balancing between the cathode and anode electrode material. Charge $(\mathrm{Q})$ is expressed by $\mathrm{Q}=\mathrm{Csp} \times \mathrm{m} \Delta \mathrm{V}$, where $\Delta \mathrm{V}$ is the working potential window.

In order to get the charge balance $\left(\mathrm{Q}^{+}=\mathrm{Q}^{-}\right)$, the mass balancing equation follows this equation $3 .^{3}$

$$
\frac{m+}{m-}=\frac{C s-\Delta v-}{C s+\Delta v+}
$$

In the case of asymmetrical supercapacitor, the specific capacitance has been estimated from CV, using the following equation $4 .^{4}$

$$
C s p=\int I(V) d V / v m V
$$

For Asymmetrical Supercapacitor, the specific capacitance has been calculated from the GCD measurement by following the equation $5 .^{5}$ 


$$
C_{s p}=\frac{I}{m(d V / d t)}
$$

Where $\mathrm{m}$ is total mass of electrode materials and $\mathrm{dV} / \mathrm{dt}$ is the slope of the discharge curve at constant current 'I'.

The energy density (ED) and power density (PD) performance has been calculated from CV plot, by following the equation 6 and $7 .{ }^{6}, 2$

$$
\begin{aligned}
& \text { E. D. }=\frac{1}{2} \operatorname{Csp}(\Delta V)^{2} \\
& \text { P.D. }=\frac{1}{2} \operatorname{Csp}(\Delta V) v
\end{aligned}
$$

The power density (PD) performance has been estimated from the GCD measurement using the following equation $8 .^{7}$

$$
\text { P. D. }=\frac{E D}{\mathrm{t}} \text {. }
$$

Where, $\mathrm{t}$ is the discharge time.

The leakage current (I) has been estimated from the self-discharge of the device using the following equation $9 .^{8}$

$$
\mathrm{I}=\mathrm{C} \times \mathrm{dV} / \mathrm{dt}
$$

Where $\mathrm{C}$, is the capacitance of the supercapacitor device and $\mathrm{dV} / \mathrm{dt}$ is the rate of change of voltage during self-discharge. 

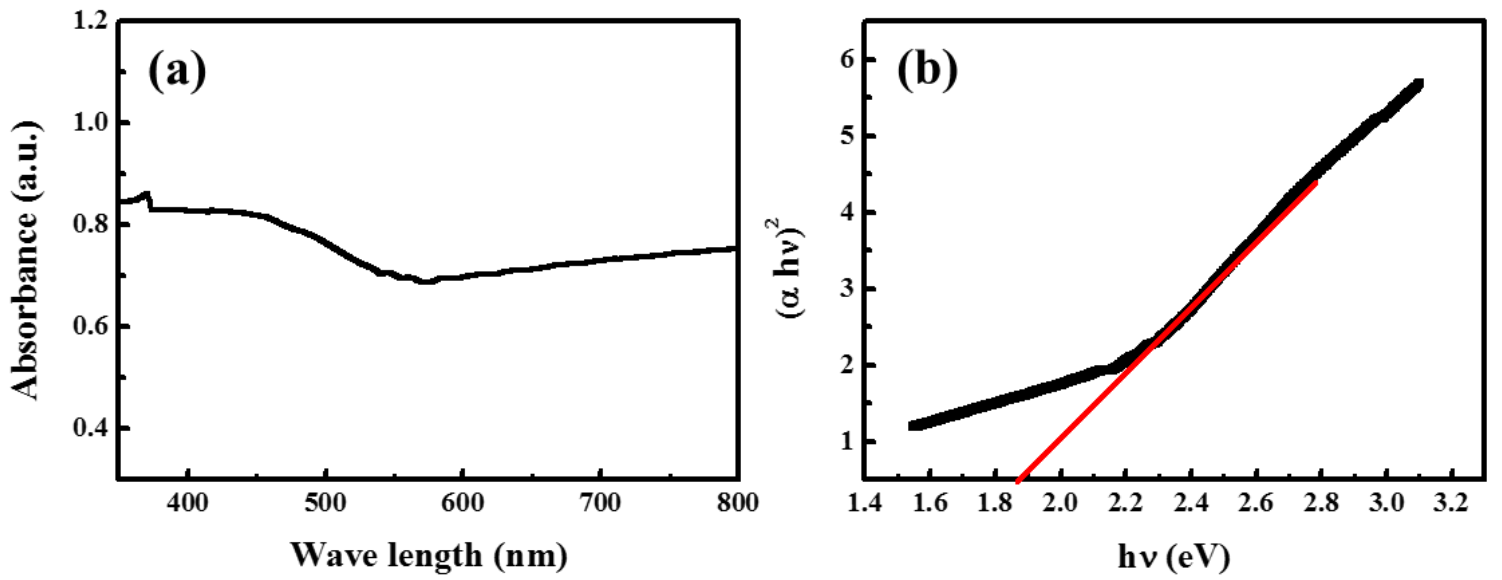

Figure S1: (a) UV-Visible absorption spectra and (b) band gap of $\mathrm{VO}_{2}(\mathrm{D})$ 

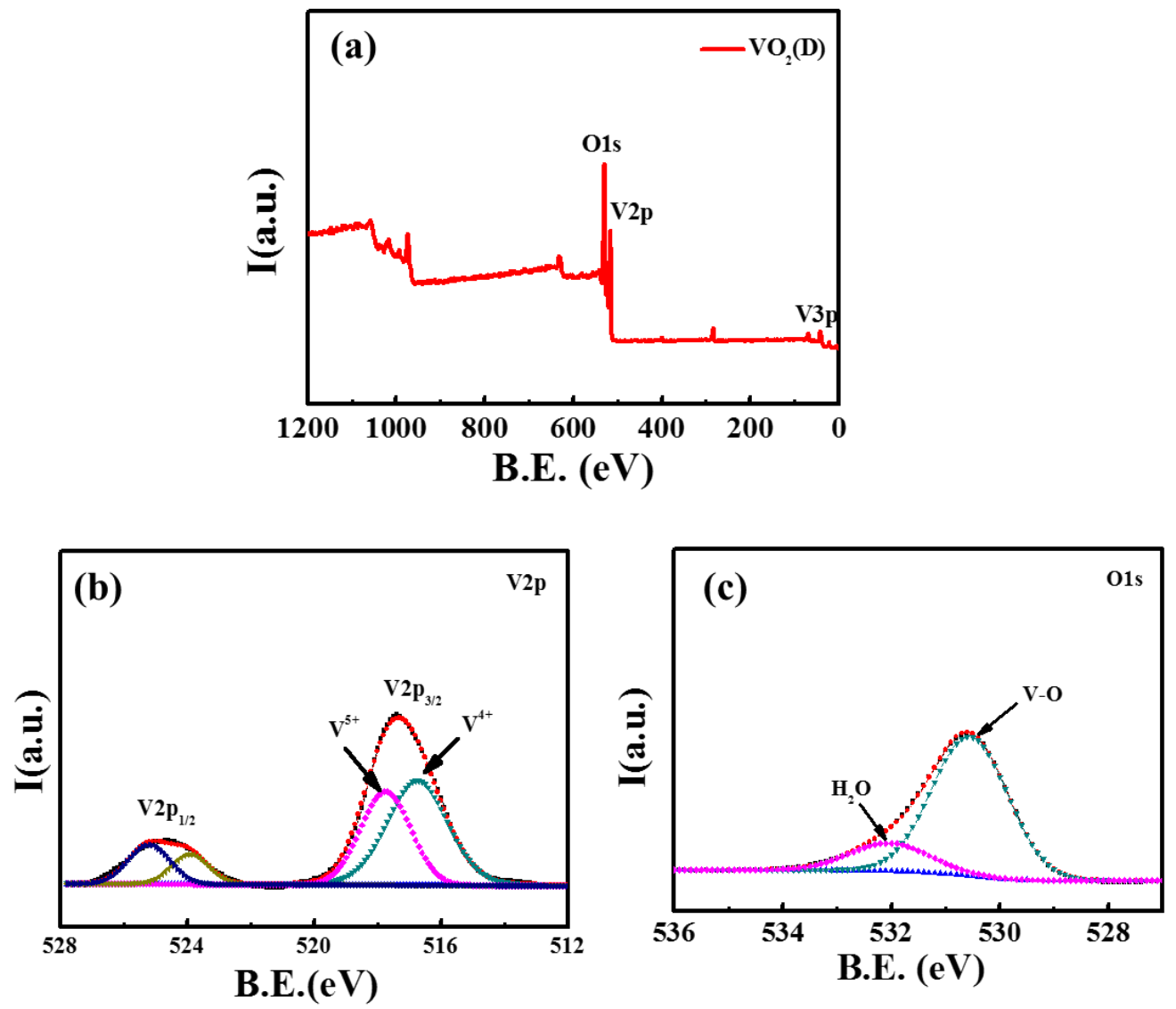

Figure S2: (a) XPS full spectrum of $\mathrm{VO}_{2}$ (D) (b) high resolution XPS spectra of V2p (c) O1s 

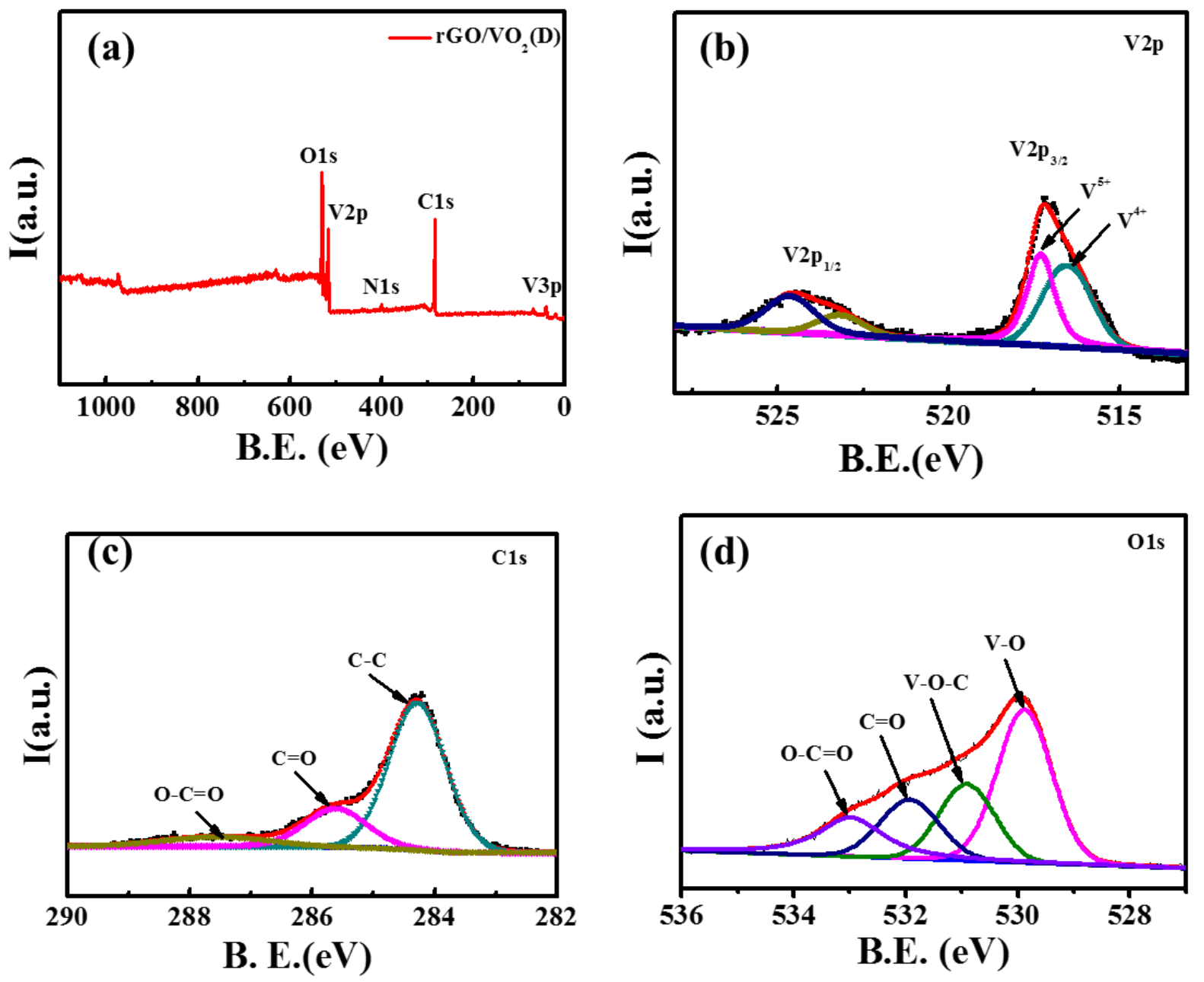

Figure S3: (a) XPS full spectrum of $\mathrm{rGO} / \mathrm{VO}_{2}$ (D) (b) high resolution XPS spectra of V2p (c) $\mathrm{C} 1 \mathrm{~s}$ and (d) $\mathrm{O} 1 \mathrm{~s}$ 

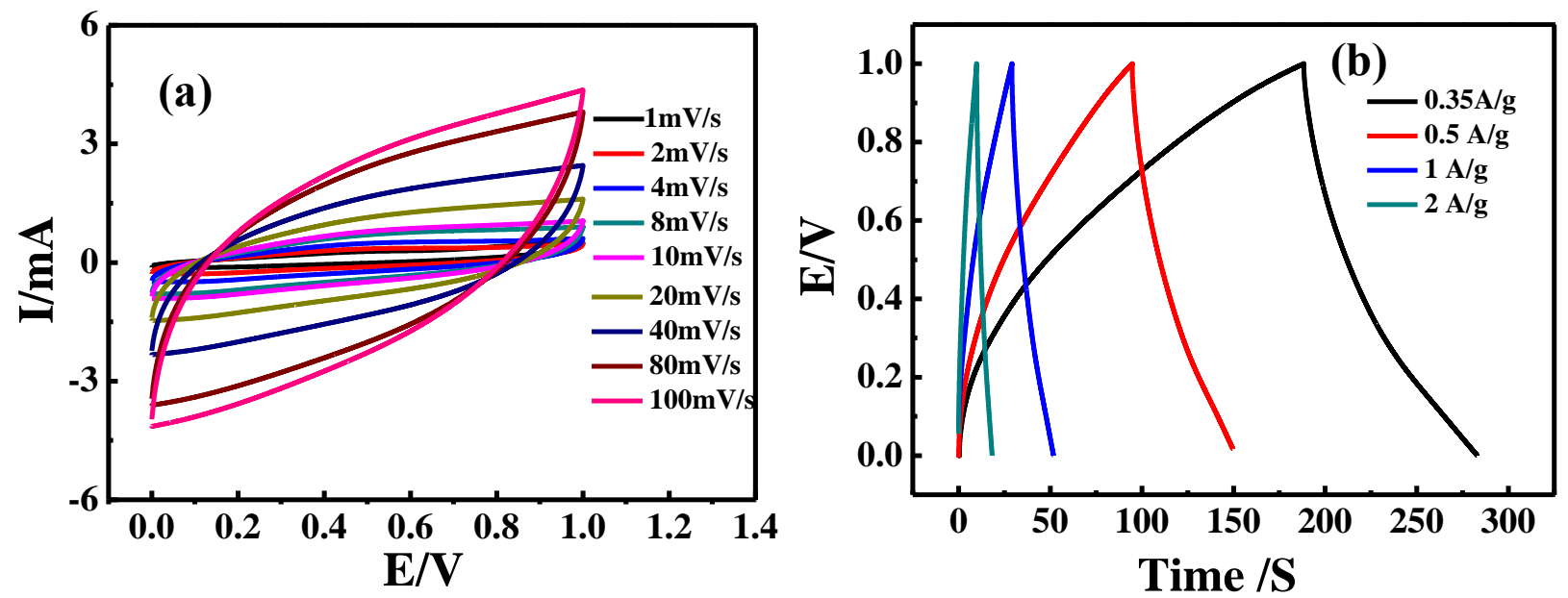

Figure S4: (a) CV plot of $\mathrm{VO}_{2}(\mathrm{D})$ at different scan rates and (b) $\mathrm{CD}$ plot of $\mathrm{VO}_{2}(\mathrm{D})$ at different current densities. 
Table S1: Performance comparison of $\mathrm{RG} / \mathrm{VO}_{2}(\mathrm{D})$ with previous reports towards supercapacitor application

\begin{tabular}{|c|c|c|c|}
\hline Materials & Specific Capacitance & Stability & References \\
\hline $\begin{array}{l}\mathrm{VO}_{2}(\mathrm{~B}) \text { nanobelts } \\
\mathrm{VO}_{2}(\mathrm{~B}) @ \text { Graphene }\end{array}$ & $\begin{array}{l}142 \mathrm{~F} / \mathrm{g} \text { at } 1 \mathrm{~A} \cdot \mathrm{g}^{-1} . \\
290.4 \mathrm{~F} / \mathrm{g} \quad 0.2 \mathrm{~A} \mathrm{~g}^{-1}\end{array}$ & $\begin{array}{l}37.9 \% 1000 \text { cycles } \\
82.3 \% 1000 \text { cycles }\end{array}$ & 9 \\
\hline $\begin{array}{l}\mathrm{VO}_{2}(\mathrm{~B}) \\
\mathrm{RG}(1.0) / \mathrm{VO}_{2}(\mathrm{~B}) \text { hybrid }\end{array}$ & $\begin{array}{l}136 \mathrm{~F} / \mathrm{g} \text { at } 0.25 \mathrm{~A} \cdot \mathrm{g}^{-1} \\
225 \mathrm{~F} / \mathrm{g} \text { at } 0.25 \mathrm{~A} \cdot \mathrm{g}^{-1} .\end{array}$ & - & 10 \\
\hline $\begin{array}{l}\text { hexangular starfruit-like } \\
\mathrm{VO}_{2}(\mathrm{~B})\end{array}$ & $216 \mathrm{~F} / \mathrm{g}$ at $1 \mathrm{~A} \cdot \mathrm{g}^{-1}$ & $95 \% 500$ cycles & 11 \\
\hline $\begin{array}{l}\mathrm{VO}_{2} \text { Nanoflower/3D } \\
\text { graphene }\end{array}$ & $507 \mathrm{~F} / \mathrm{g} 3 \mathrm{~mA} / \mathrm{cm}^{2}$ & $63.5 \% 3000$ cycles & 12 \\
\hline $\mathrm{VO}_{2}(\mathrm{~B}) / \mathrm{CNT}$ & $250 \mathrm{~F} / \mathrm{g}$ at $0.5 \mathrm{~A} / \mathrm{g}$ & $70 \% 200$ cycles & 13 \\
\hline $\mathrm{VO}_{2} / \mathrm{TiO}_{2}$ Nanosponges & $548 \mathrm{~F} / \mathrm{g}$ at $10 \mathrm{mV} / \mathrm{s}$ & $84.3 \% 1000$ cycles & 14 \\
\hline $\mathrm{VO}_{2}(\mathrm{~B}) / \mathrm{GN}$ & $197 \mathrm{~F} / \mathrm{g}$ at $1 \mathrm{~A} / \mathrm{g}$ & $95.49 \% 1000$ cycles & 15 \\
\hline $2 \mathrm{D}$ mesoporous $\mathrm{VO}_{2}$ & $265 \mathrm{~F} / \mathrm{g} 1 \mathrm{~A} / \mathrm{g}$ & $\begin{array}{l}\text { No capacitance loss, } \\
3000 \text { cycles }\end{array}$ & 16 \\
\hline $\begin{array}{l}\mathrm{VO}_{2}(\mathrm{~B}) / \text { mesoporous } \\
\text { carbon }\end{array}$ & $131 \mathrm{~F} / \mathrm{g}$ at $0.2 \mathrm{~A} / \mathrm{g}$ & - & 17 \\
\hline $\begin{array}{l}\mathrm{VO}_{2}(\mathrm{~B}) / \text { Carbon } \\
\text { composite }\end{array}$ & $182 \mathrm{~F} / \mathrm{g}$ at $1 \mathrm{~A} / \mathrm{g}$ & $10.4 \% 100$ cycles & 18 \\
\hline ellipsoidal $\mathrm{VO}_{2}$ & $60 \mathrm{~F} / \mathrm{g}$ at $0.25 \mathrm{~A} / \mathrm{g}, 1.6 \mathrm{~V}$ & $78 \% 4500$ cycles & 19 \\
\hline $\begin{array}{l}\mathrm{VO}_{2} \text { Nanobelt } \\
3 \mathrm{D} \text { graphene/ } \mathrm{VO}_{2} \\
\text { nanobelt }\end{array}$ & $\begin{array}{l}191 \mathrm{~F} / \mathrm{g} \text { at } 1 \mathrm{~A} / \mathrm{g} \\
426 \mathrm{~F} / \mathrm{g} \text { at } 1 \mathrm{~A} / \mathrm{g}\end{array}$ & $\begin{array}{l}59 \% 5000 \text { cycles } \\
92 \% 5000 \text { cycles }\end{array}$ & 20 \\
\hline $\mathrm{VO}_{2}(\mathrm{D})$ plates & $\begin{array}{l}271 \mathrm{~F} / \mathrm{g} \text { at } 1 \mathrm{mV} / \mathrm{s} \\
46 \mathrm{~F} / \mathrm{g} \text { at } 1 \mathrm{~A} / \mathrm{g}\end{array}$ & $59 \% 5000$ cycles & This work \\
\hline $\mathrm{rGO} / \mathrm{VO}_{2}(\mathrm{D})$ hybrids & $\begin{array}{l}737 \mathrm{~F} / \mathrm{g} \text { at } 1 \mathrm{mV} / \mathrm{s} \\
244 \mathrm{~F} / \mathrm{g} \text { at } 1 \mathrm{~A} / \mathrm{g}\end{array}$ & $\begin{array}{l}\text { No capacitance loss } \\
5000 \text { cycle }\end{array}$ & This work \\
\hline
\end{tabular}



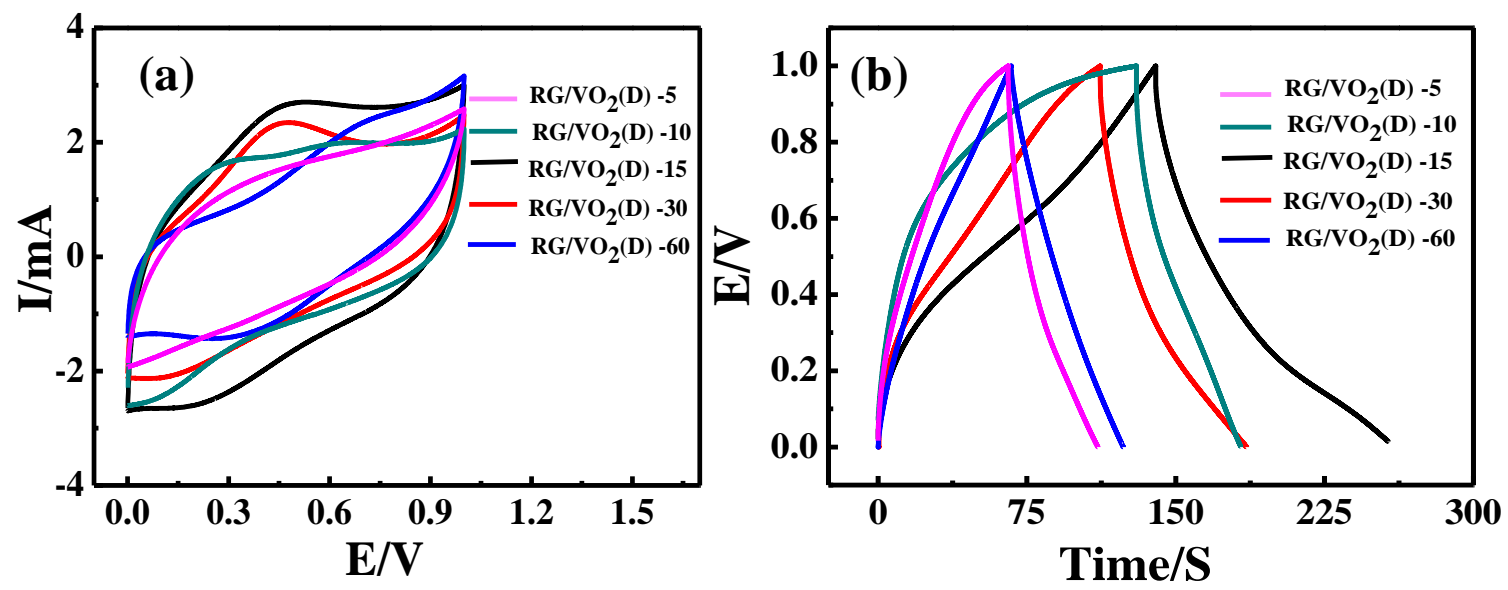

Figure S5: Overlap (a) $\mathrm{CV}$ at $10 \mathrm{mV} / \mathrm{s}$ and (b) $\mathrm{GCD}$ at $1 \mathrm{~A} / \mathrm{g}$ at different concentration of $\mathrm{RG}$ in $\mathrm{rGO} / \mathrm{VO}_{2}(\mathrm{D})$ hybrid. 

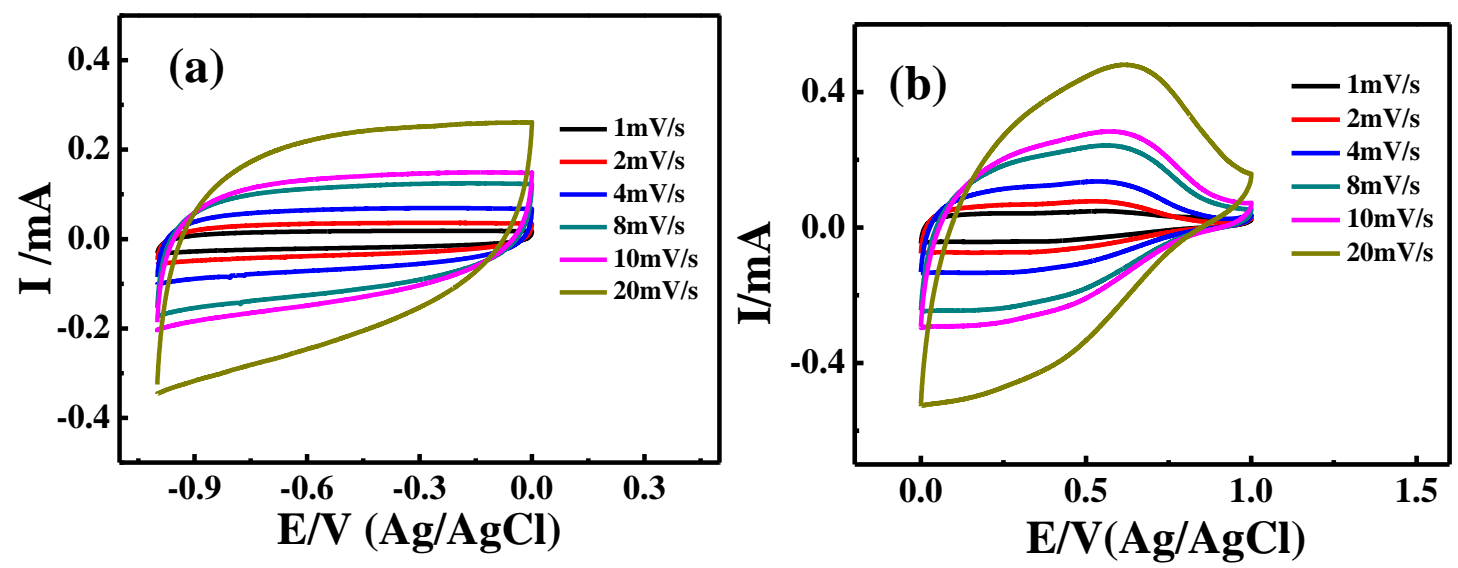

Figure S6: (a) $\mathrm{CV}$ curves of $\mathrm{rGO}$ and (b) $\mathrm{CV}$ curves of $\mathrm{rGO} / \mathrm{VO}_{2}(\mathrm{D})$ at different scan rates. 

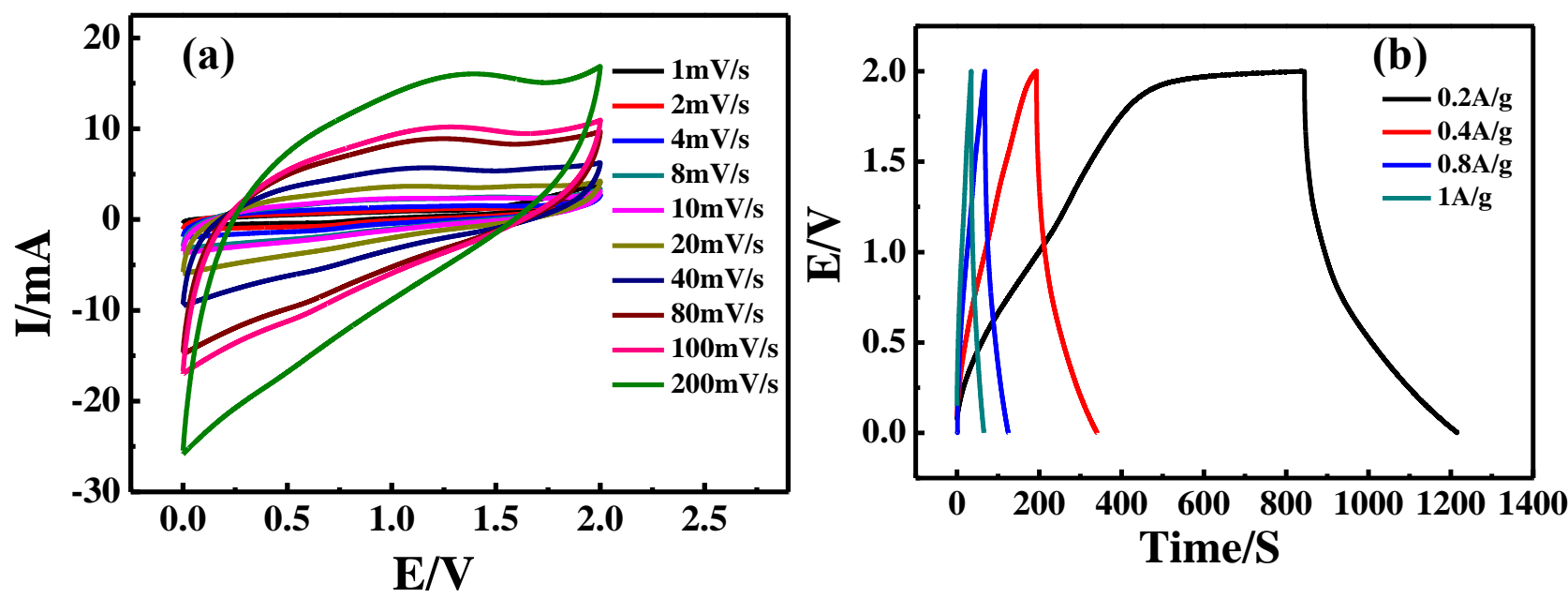

Figure S7: (a) CV curves at different scan rates and (b) CD curves at different current densities of asymmetric coin cell supercapacitor 

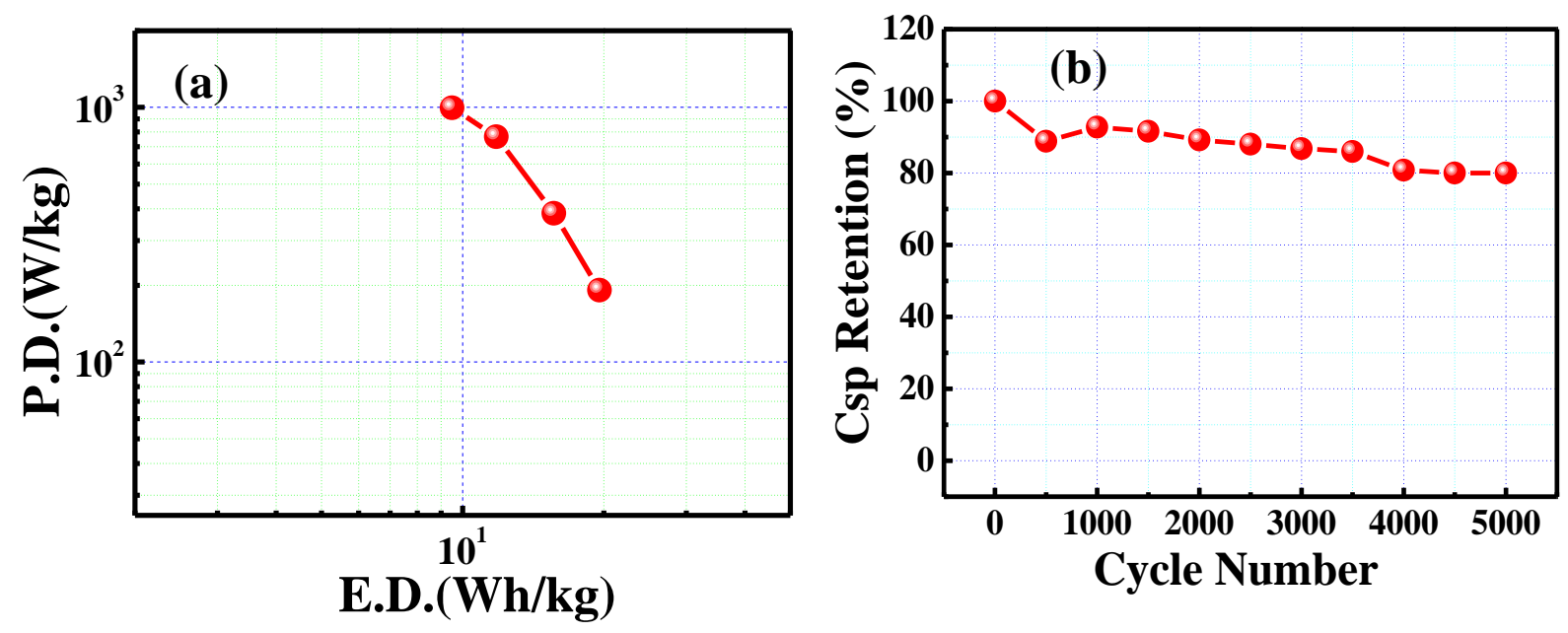

Figure S8: (a) Ragone Plot and (b) Operational stability of asymmetric coin cell supercapacitor 


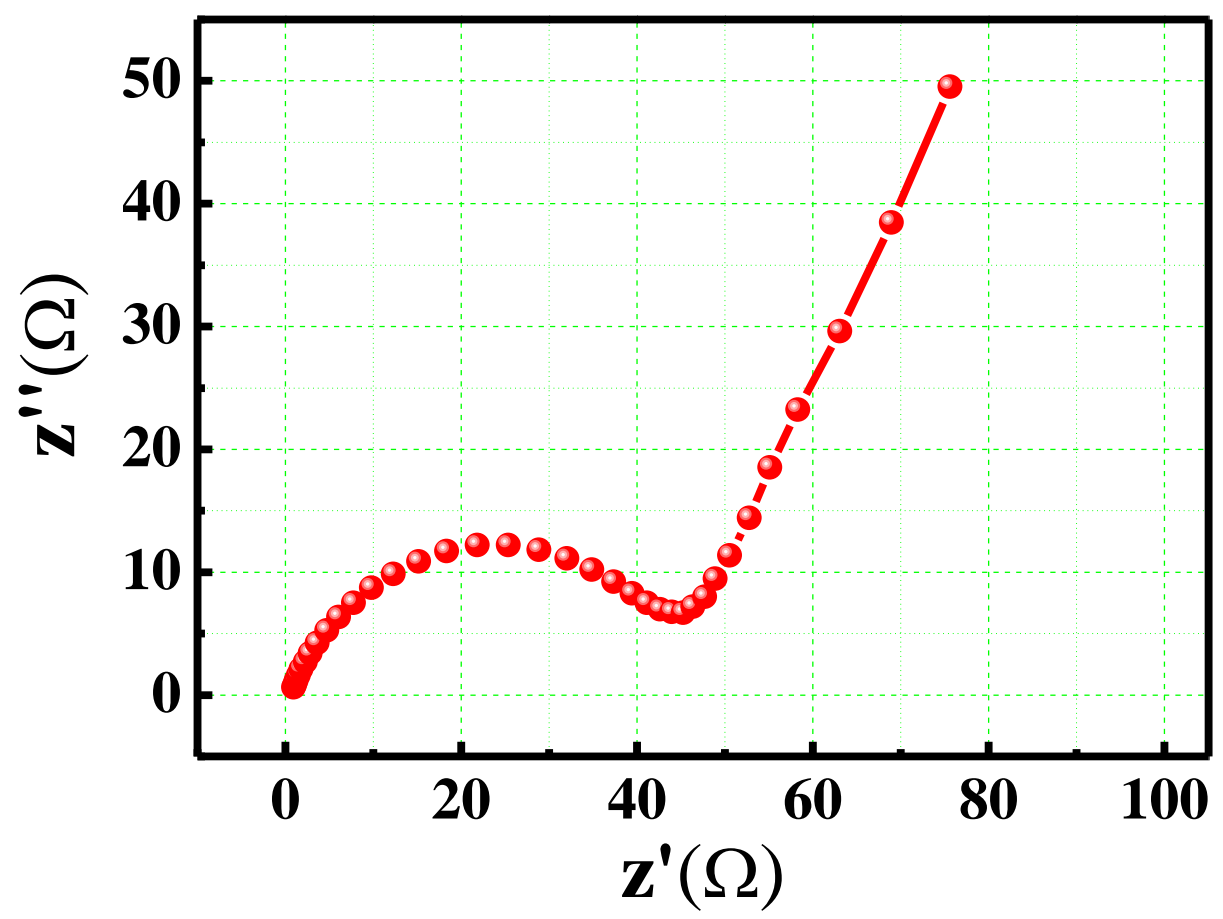

Figure S9: EIS Nyquist plot of the as-fabricated coin-cell asymmetric device. 

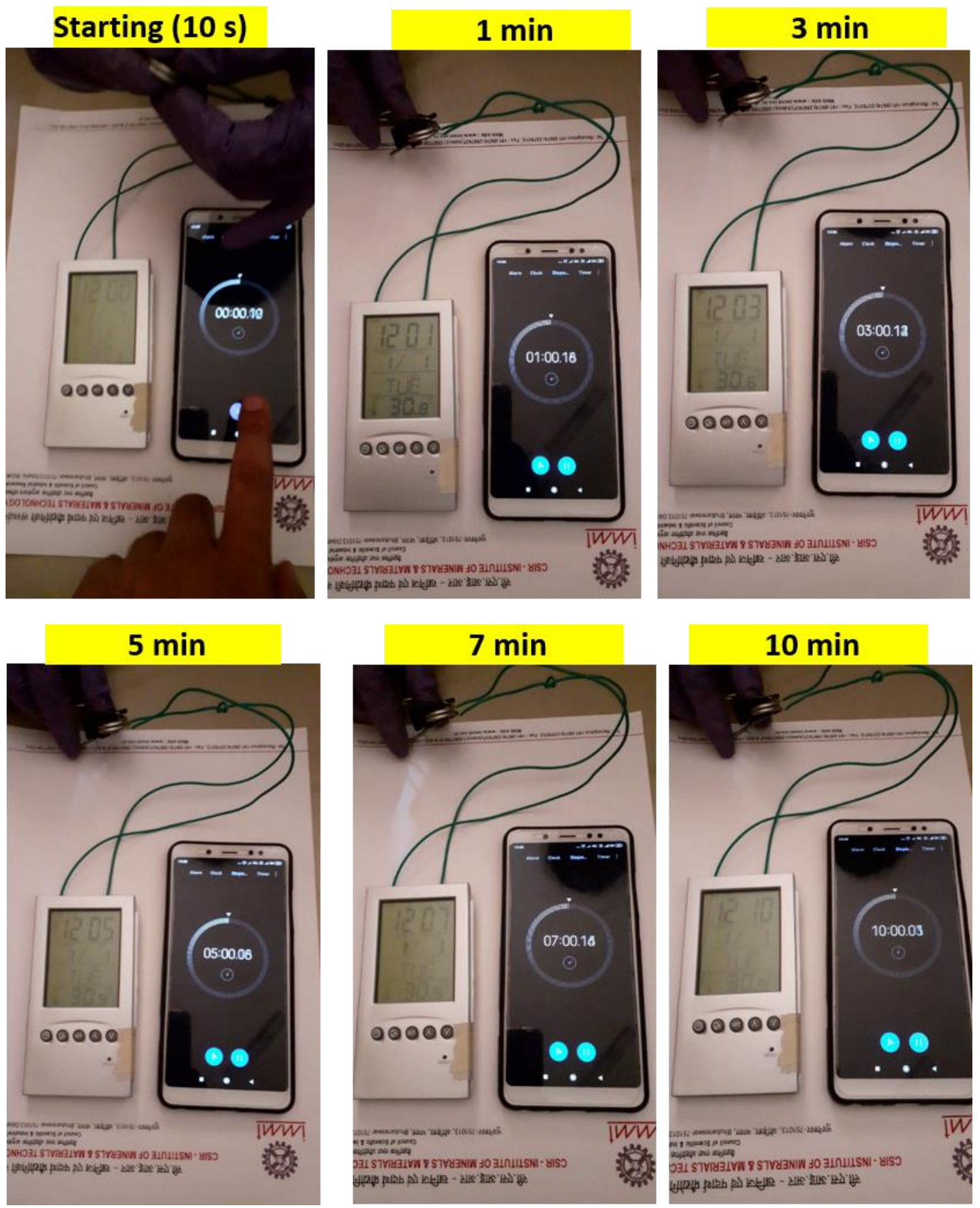

Figure S10: Snapshots of as-fabricated asymmetric coin cell device showing the powering of a digital watch at different duration of time. 

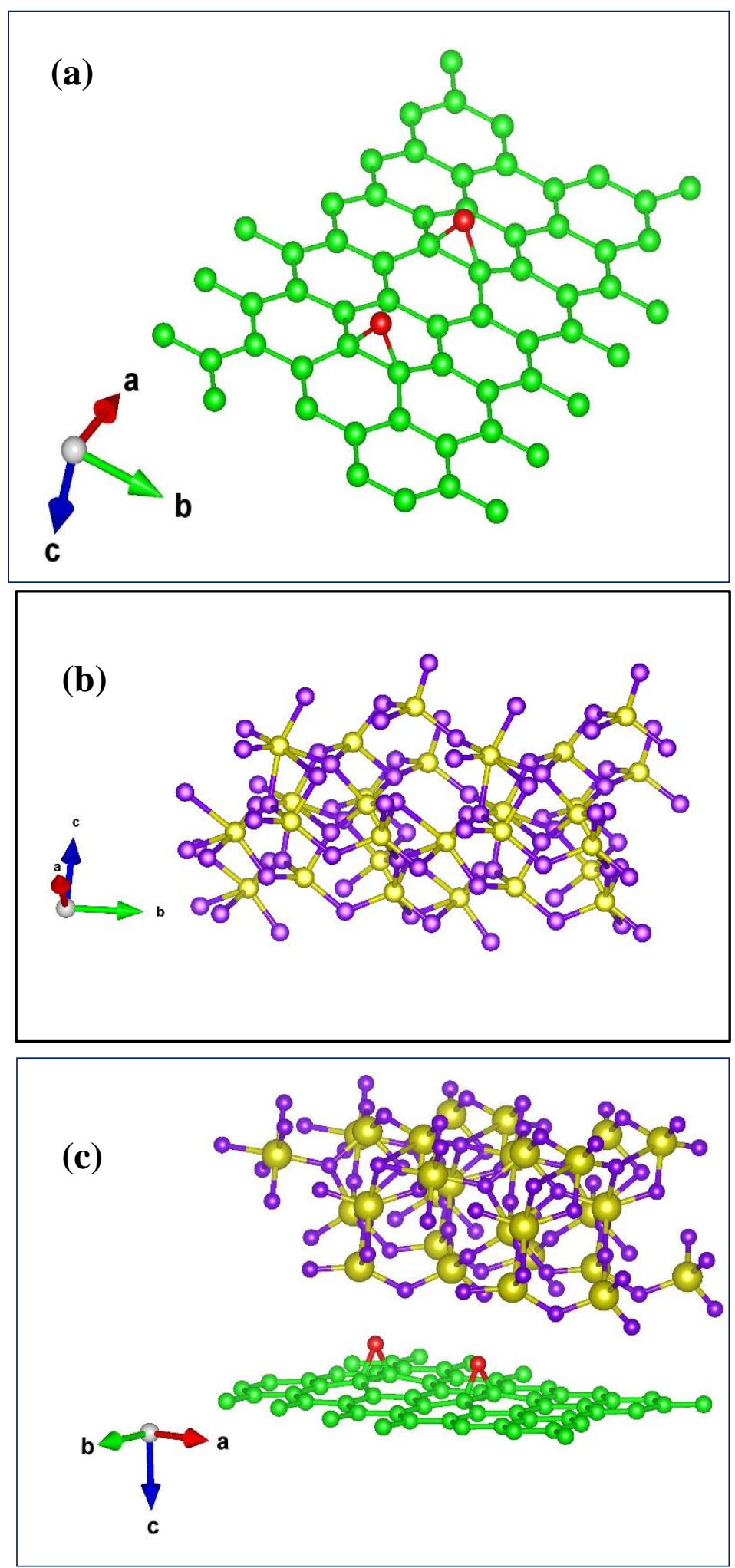

Figure S11: DFT optimized structures for (a) rGO, (b) (011) surface of $\mathrm{VO}_{2}(\mathrm{D})$, (c) Hybrid structure of (011) surface of $\mathrm{VO}_{2}(\mathrm{D})$ and $\mathrm{rGO}$; green, purple and golden sphere indicates $\mathrm{C}, \mathrm{O}$ and $\mathrm{V}$ atoms respectively. Red sphere indicate $\mathrm{O}$ of epoxy group. 


\section{References}

(1) Kamila, S.; Mohanty, B.; Samantara, A. K.; Guha, P.; Ghosh, A.; Jena, B.; Satyam, P. V; Mishra, B. K.; Jena, B. K. Highly Active 2D Layered MoS(2)-RGO Hybrids for Energy Conversion and Storage Applications. Scientific Reports 2017, 7, 8378.

(2) Samantara, A. K.; Chandra Sahu, S.; Ghosh, A.; Jena, B. K. Sandwiched Graphene with Nitrogen, Sulphur Co-Doped CQDs: An Efficient Metal-Free Material for Energy Storage and Conversion Applications. J. Mater. Chem. A 2015, 3 (33), 16961-16970.

(3) Wang, J.; Shen, L.; Nie, P.; Yun, X.; Xu, Y.; Dou, H.; Zhang, X. N-Doped Carbon Foam Based Three-Dimensional Electrode Architectures and Asymmetric Supercapacitors. Journal of Materials Chemistry A 2015, 3 (6), 2853-2860.

(4) Yan, J.; Fan, Z.; Sun, W.; Ning, G.; Wei, T.; Zhang, Q.; Zhang, R.; Zhi, L.; Wei, F. Advanced Asymmetric Supercapacitors Based on Ni(OH)2/Graphene and Porous Graphene Electrodes with High Energy Density. Advanced Functional Materials 2012, 22 (12), 26322641.

(5) Lu, X.; Zeng, Y.; Yu, M.; Zhai, T.; Liang, C.; Xie, S.; Balogun, M.-S.; Tong, Y. OxygenDeficient Hematite Nanorods as High-Performance and Novel Negative Electrodes for Flexible Asymmetric Supercapacitors; 2014; Vol. 26.

(6) Tabassum, H.; Mahmood, A.; Wang, Q.; Xia, W.; Liang, Z.; Qiu, B.; zhao, R.; Zou, R. Hierarchical Cobalt Hydroxide and B/N Co-Doped Graphene Nanohybrids Derived from Metal-Organic Frameworks for High Energy Density Asymmetric Supercapacitors. Scientific Reports 2017, 7, 43084.

(7) Anothumakkool, B.; Torris A. T, A.; Bhange, S. N.; Badiger, M. V; Kurungot, S. Electrodeposited Polyethylenedioxythiophene with Infiltrated Gel Electrolyte Interface: A Close Contest of an All-Solid-State Supercapacitor with Its Liquid-State Counterpart. Nanoscale 2014, 6 (11), 5944-5952.

(8) Dinglasan-Fenol, S.; Caluyo, F. S.; Sugimoto, W. Effect of Charging Methods on SelfDischarge and Leakage Current of Supercapacitors. In 2016 4th International Conference on Control Engineering \& Information Technology (CEIT); 2016; pp 1-5.

(9) Xiao, X.; Li, S.; Wei, H.; Sun, D.; Wu, Y.; Jin, G.; Wang, F.; Zou, Y. Synthesis and Characterization of VO2(B)/Graphene Nanocomposite for Supercapacitors. Journal of Materials Science: Materials in Electronics 2015, 26, 4226-4233.

(10) Deng, L.; Zhang, G.; Kang, L.; Lei, Z.; Liu, C.; Liu, Z.-H. Graphene/VO2 Hybrid Material for High Performance Electrochemical Capacitor. Electrochimica Acta 2013, 112, 448-457.

(11) Shao, J.; Li, X.; Qu, Q.; Zheng, H. One-Step Hydrothermal Synthesis of Hexangular Starfruit-like Vanadium Oxide for High Power Aqueous Supercapacitors. Journal of Power Sources 2012, 219, 253-257.

(12) Wang, J.; Zhang, X.; Zhang, Y.; Abas, A.; Zhao, X.; Yang, Z.; Su, Q.; Lan, W.; Xie, E. Lightweight, Interconnected VO2 Nanoflowers Hydrothermally Grown on 3D Graphene 
Networks for Wide-Voltage-Window Supercapacitors. RSC Advances 2017, 7 (56), 3555835564.

(13) Liang, L.; Liu, H.; Yang, W. Fabrication of VO2(B) Hybrid with Multiwalled Carbon Nanotubes to Form a Coaxial Structure and Its Electrochemical Capacitance Performance; 2013; Vol. 559.

(14) Hu, C.; Xu, H.; Liu, X.; Zou, F.; Qie, L.; Huang, Y.; Hu, X. VO2/TiO2 Nanosponges as Binder-Free Electrodes for High-Performance Supercapacitors. Scientific Reports 2015, 5 , 16012 .

(15) Zhang, J.; Chen, L.; Wang, Y.; Cai, S.; Yang, H.; Yu, H.; Ding, F.; Huang, C.; Liu, X. VO2(B)/Graphene Composite-Based Symmetrical Supercapacitor Electrode via Screen Printing for Intelligent Packaging. Nanomaterials . 2018.

(16) Fan, Y.; Ouyang, D.; Li, B.-W.; Dang, F.; Ren, Z. Two-Dimensional VO2 Mesoporous Microarrays for High-Performance Supercapacitor. Nanoscale Research Letters 2018, 13 (1), 142.

(17) Hu, L.; Yu, L.; Zhao, C.; Long, X.; Chen, W. Synthesis and Characterization of VO2/Mesoporous Carbon Composites for Hybrid Capacitors. Journal of Wuhan University of Technology-Mater. Sci. Ed. 2010, 25 (4), 574-578.

(18) Zhang, Y.; Zheng, J.; Hu, T.; Tian, F.; Meng, C. Synthesis and Supercapacitor Electrode of VO2(B)/C Core-Shell Composites with a Pseudocapacitance in Aqueous Solution. Applied Surface Science 2016, 371, 189-195.

(19) Ma, X.-J.; Zhang, W.-B.; Kong, L.-B.; Luo, Y.-C.; Kang, L. VO2: From Negative Electrode Material to Symmetric Electrochemical Capacitor. RSC Advances 2015, 5 (118), 9723997247.

(20) Wang, H.; Yi, H.; Chen, X.; Wang, X. One-Step Strategy to Three-Dimensional Graphene/VO2 Nanobelt Composite Hydrogels for High Performance Supercapacitors. Journal of Materials Chemistry A 2014, 2 (4), 1165-1173. 